\title{
A Two-phase Algorithm for Locating Sensors in Irregular Areas
}

\author{
Wenbo Shi and Vincent W.S. Wong \\ Department of Electrical and Computer Engineering, \\ The University of British Columbia, Vancouver, Canada \\ E-mail: $\{$ wenbos, vincentw $\} @$ ece.ubc.ca
}

\begin{abstract}
In wireless sensor networks, location-aware applications require an accurate and robust sensor localization algorithm. Among them, most of the multihop-based localization algorithms approximate the shortest path distances to the Euclidean distances. This approximation is valid only if the sensors are uniformly and densely deployed in a convex area where the shortest paths are close to straight lines. However, in a real-world setting, the convexity assumption may not always be valid. Non-convex deployment areas, such as $\mathrm{C}$-shaped or S-shaped topologies, can corrupt the localization results severely due to erroneous distance estimations distorted by the non-convex topology. In this paper, we formulate the localization problem in irregular areas as a constrained least-penalty problem. We then propose a two-phase algorithm to eliminate the impact of irregularities. In the first phase, the estimated position is confined in the intersection area of the communication range constraints. In the second phase, the distorted measurements are eliminated by using a robust position estimator. Simulation results show that the two-phase algorithm outperforms some of the existing multihop localization algorithms in terms of a lower average localization error in both $\mathrm{C}$-shaped and $\mathrm{S}$-shaped topologies. The effects of anchor density, range error and communication range on localization performances are studied as well.
\end{abstract}

\section{INTRODUCTION}

Localization is a crucial issue in wireless sensor networks as it provides support for different types of location-aware applications (e.g., position-based routing [1], monitoring, target tracking). One way to acquire location information is to equip each sensor with a global positioning system (GPS). However, this approach may not be cost effective for large scale deployment [2]. In practice, only a limited proportion of sensors, which are called anchors, are equipped with GPS. The distributed localization problem for each sensor is to estimate its position based on its relative distances to some of those anchors. Various centralized [3], [4] and distributed localization algorithms [5], [6] have been proposed for wireless sensor networks. They can further be classified into range-based [3], [7], angle-based [8], [9], and range-free [10], [11] approaches. Distributed algorithms have advantages over centralized schemes with respect to scalability and computational efficiency. Range-based approaches can produce better estimates than angle-based and range-free schemes. In this paper, we mainly focus on distributed range-based localization.

Range-based localization schemes assume that the sensor has the ability to obtain distance measurements to neighboring nodes [2]. The distance information can be gathered by a variety of ways including the use of received signal strength
(RSS) [12], time-of-arrival (TOA) [13], and time-differenceof-arrival (TDOA) [14]. In wireless sensor networks, the ranging equipment can achieve desirable accuracy only in a short-range due to cost and energy constraints. Multihop-based localization approaches [2], which are based on the assumption that the shortest path distances are close to the Euclidean distances, are proposed to deal with the limitation of the shortrange problem. However, the Euclidean distance can only be well approximated if the shortest path is a non-distorted path with line of sight, which requires the sensors to be densely and uniformly distributed in a convex area [15]. Such an assumption does not hold if the deployment area is irregular. The shortest path between some nodes may be distorted by the non-convex shape. Under such circumstances, the results of multihop-based algorithms can be severely corrupted.

In this paper, we study the problem of locating sensors in irregular areas. Our contributions are as follows:

- We propose a two-phase algorithm to estimate the positions of sensors in non-convex areas. The localization problem is formulated as a least-penalty problem with communication range constraints.

- We minimize the impact of irregularities in localization through two phases. In the first phase, the potential area of the sensor is determined by the intersection of the communication range constraints from anchors. In the second phase, the distorted paths are excluded from localization by using a robust position estimator.

- We conduct simulations to evaluate the performance of our proposed two-phase algorithm and compare it with some existing multihop algorithms [15], [16]. Results show that the proposed algorithm can effectively eliminate the impact of irregularities and outperforms the other two algorithms in C-shaped and S-shaped topologies.

- We evaluate our two-phase algorithm under different anchor density, range error and communication range. Based on simulation results, we provide the conditions under which the two-phase algorithm has a significant gain over other existing multihop algorithms [15], [16].

The rest of this paper is organized as follows. The related localization techniques are summarized in Section II. Our proposed two-phase algorithm is presented in Section III. Performance evaluation and comparison are presented in Section IV. Conclusions are given in Section V. 


\section{RELATED WORK}

One of the first multihop localization algorithms is the ad hoc positioning system (APS) proposed in [2]. For the range-based scheme which refers to the DV-distance, each node has the ability to measure the distances to neighboring nodes. The anchors first announce their locations to all sensors via flooding and then each sensor uses multilateration to estimate its position. The work in [15] showed that localization results of multihop algorithms can be severely corrupted by irregular deployment areas. To deal with the erroneous distance measurements due to the irregular topologies, it is suggested to use the four nearest anchors instead of all of the anchors for multilateration in [16]. The rationale is that it is less likely for the shortest paths to the nearest anchors to be affected by the irregular shape. This scheme can produce good localization results in some cases. However, using only four nearest anchors for localization may ignore some other potential good distance measurements which can be utilized to improve the accuracy.

An improved multihop localization algorithm called $i$ Multihop: hybrid approach was proposed in [15]. The algorithm estimates the position of a sensor to be within the intersection area of all the circles from the anchors using a leastsquares estimator. However, since the estimated position of the sensor is calculated by using the distance measurements of all anchors, the impact of the distorted paths to anchors cannot be totally eliminated. A range-free multihop scheme named reliable anchor-based localization (RAL) [17] was proposed to eliminate the impact of irregular deployment areas. They used the minimum hop length from a pre-constructed table to filter out unreliable anchors distorted by non-convex shapes. The position of each sensor is estimated using the distance information only from the reliable anchors. RAL is a rangefree scheme and is not applicable to range-based localization in irregular areas.

Most of the current localization schemes use the leastsquares estimator to calculate the estimated position. However, it is well known that the least-squares estimator is not robust. The estimated result can be biased even when there are only a few noisy measurements with the rest being accurate [18]. In order to filter out the outliers in the context of localization, Kung et al. [19] proposed an error-tolerant localization scheme called snap-inducing shaped residuals (SISR), which uses a residual shaping function to snap into the true positions regardless of the bad measurements. SISR is a centralized localization scheme and it uses multidimensional scaling (MDS$\operatorname{MAP}(C, R))[3]$ as the initial approximation.

This paper differs from the existing related work in the literature in several aspects. Unlike the four nearest anchors algorithm in [16], which uses limited distance information to the nearest anchors, here we solve the localization problem based on all reliable distance measurements with data redundancy to improve the accuracy. Using communication range constraints in localization, which refers to the first phase in our two-phase algorithm, has also been addressed in [15], [20]. However, neither the rectangle bound in [20] nor the least-squares fitting in [15] performs well when the position of the sensor cannot be tightly bounded by the intersection of communication range constraints. Our proposed two-phase algorithm solves this problem by estimating the position within the intersection area based on reliable measurements in the second phase. It is also worth mentioning that the idea of localization based only on reliable measurements can be found in RAL [17] and SISR [19]. However, our work here addresses distributed rangebased localization, which is more scalable and computational efficient than the centralized algorithm [19]. Our scheme is more accurate compared with the range-free scheme [17]. Last but not least, the mathematical technique that we use in our proposed two-phase algorithm to filter out outliers has its roots in robust statistics [21]. However, applying similar techniques in the context of distributed localization is novel.

\section{Two-PHASE LOCALIZATION ALGORITHM}

In this section, we describe our proposed two-phase sensor localization algorithm in detail. We relax the convexity assumption in multihop-based algorithms. Sensors can be distributed in any deployment areas. All anchors are equipped with GPS and know their true positions. Each sensor has the ability to measure its distances to neighboring nodes using ranging techniques such as TOA or ultrasonic.

Our proposed sensor localization scheme is carried out by first propagating the anchor information and distance measurements using a shortest path algorithm. When each sensor obtains the shortest path information to all anchors, the twophase algorithm is performed at each sensor to estimate its position. The algorithm has two consecutive phases:

1) The determination of the potential area where the sensor is located;

2) The estimation of position which discards erroneous measurement distorted by the non-convex shape.

We define $\hat{d}_{s a}$ to be the shortest path length from sensor $s \in \mathcal{S}$ to anchor $a \in \mathcal{A}$ where $\mathcal{A}$ denotes the set of anchors and $\mathcal{S}$ denotes the set of sensors. $C_{a}$ defines the communication area of anchor $a . P_{s}$ represents the potential area of sensor $s$. Algorithm 1 presents the pseudocodes for the localization algorithm executed on each sensor $s \in \mathcal{S}$. In Algorithm 1, lines 2 to 11 refer to the shortest path algorithm which are executed repeatedly until the flooding is completed. In lines 4 to 6 , sensor $s$ finds its shortest path distance to anchor $a$ either via node $i$ or another node. Node $i$ can be either a sensor or an anchor. Sensor $s$ updates its shortest path distance to anchor $a$ if $\hat{d}_{s i}+\hat{d}_{i a}<\hat{d}_{s a}$, where $\widehat{d}_{s i}$ is the distance between sensor $s$ and node $i$ obtained by the range equipment. In lines 7 to 9 , sensor $s$ broadcasts its shortest path lengths to all neighboring sensors and anchors if any of them is updated. Lines 12 and 13 refer to the two phases for locating sensor $s$ respectively.

\section{A. Phase 1: Potential Area Approximation}

In the first phase, the objective is to estimate the position by identifying the potential area of sensor $s \in \mathcal{S}$. We model the radio frequency communication area to be a circular 


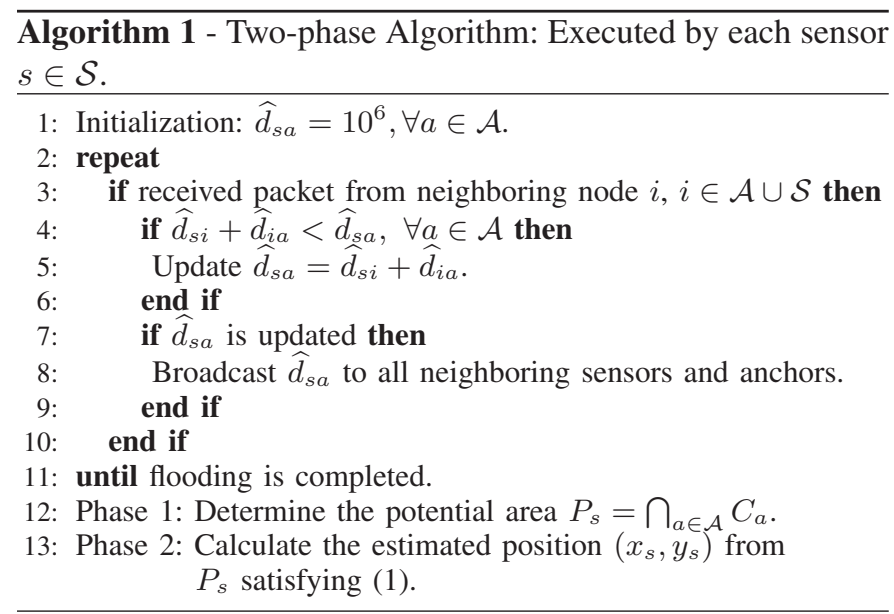

area. This is not an accurate physical representation since the actual shape of the area is often anisotropic and time-varying. However, our proposed two-phase algorithm can be easily applied to other communication models (e.g. ellipses). Given the distance measurement $\hat{d}_{s a}$ between sensor $s$ and anchor $a$, we assume that sensor $s$ must be within the communication area of anchor $a$, which refers to the circle originated from anchor $a$ with radius of $\hat{d}_{s a}$. With more distance measurements to anchors, the position of sensor $s$ can be bounded in the intersection of the communication range constraints from all anchors $P_{s}=\bigcap_{a \in \mathcal{A}} C_{a}$.

Fig. 1 illustrates how the first phase filters out the distorted path. In this figure, four anchors $a_{1}$ to $a_{4}$ and five sensors $s_{1}$ to $s_{5}$ are deployed. We consider the localization problem for sensor $s_{5}$, which is within the one hop range of anchors $a_{1}$ to $a_{3}$ but five hops from $a_{4}$. We can see that the shortest path from anchor $a_{4}$ to sensor $s_{5}$ is distorted by the obstacle. Thus the distance measurement $\hat{d}_{s_{5} a_{4}}$ has a relative large value while the other measurements are close to their Euclidean distances. The potential area of sensor $s_{5}$ is approximated as the intersection $\bigcap_{a \in \mathcal{A}} C_{a}$ shown as the shaded area in the figure which is determined by the circular communication ranges from only anchors $a_{1}$ to $a_{3}$. The large radius of anchor $a_{4}$ does not affect the potential area of sensor $s_{5}$. Let $d_{s a}$ denote the Euclidean distance between sensor $s$ and anchor $a$. Depending on reliable distance measurements $\hat{d}_{s a} \approx d_{s a}$, the position of the sensor can be well approximated in the intersection area of the circular communication range constraints. Note that the intersection area is a good approximation of the sensor's position only if the anchors are not all on the same side of the sensor. Otherwise, the area $P_{s}=\bigcap_{a \in \mathcal{A}} C_{a}$ cannot tightly bound the position of the sensor giving an unreliable approximation. An illustration of such cases is shown in Fig. 2. In the figure, it can be seen that the position of sensor $s$ cannot be well approximated by the intersection area $P_{s}$ since all three anchors are on the left side of sensor $s$.

When the distance measurements are accurate, the shortest path length $\hat{d}_{s a}$ is always overestimated, i.e., $\hat{d}_{s a} \geq d_{s a}$. Thus the intersection of the communication areas from all anchors

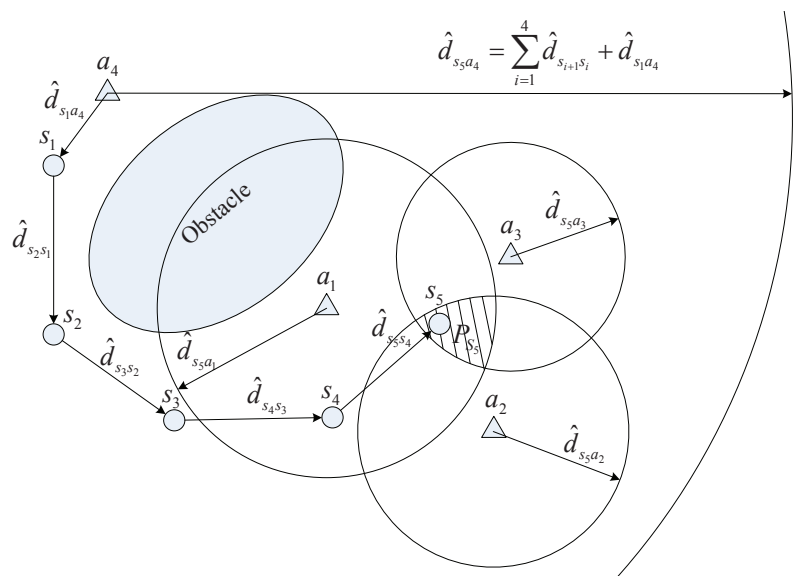

Fig. 1. An illustration of the potential area of sensor $s_{5}$, i.e., $P_{s_{5}}$ (shaded area). Anchors are represented by triangles, sensors are plotted as circles. Distorted path from $a_{4}$ does not affect $P_{s_{5}}$.

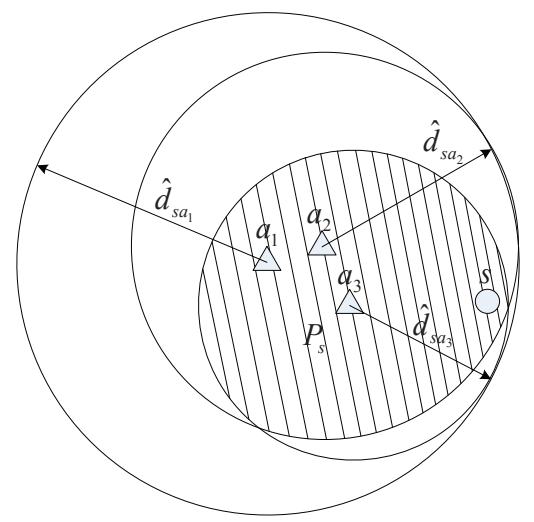

Fig. 2. An illustration of the potential area of sensor $s$ when anchors are all on the same side of the sensor, i.e., $P_{s}$ (shaded area). Anchors are represented by triangles, sensor $s$ is plotted as a circle. The position of sensor $s$ cannot be well approximated by $P_{s}$.

$P_{s}=\bigcap_{a \in \mathcal{A}} C_{a}$ always exists. However, in the presence of range errors, the shortest path length may be smaller than the Euclidean distance and the circular communication ranges from anchors may not intersect with each other. In such cases, the feasible set of the localization problem does not exist. When small range errors exist, we can add a slack variable $\varepsilon_{s a} \geq 0$ to the distance measurement $\hat{d}_{s a}$ to compensate the negative range error. Thus the potential area of sensor $s$ becomes $P_{s}=\bigcap_{a \in \mathcal{A}} C_{a}$ where $C_{a}$ denotes the circular communication area from anchor $a$ with radius of $\hat{d}_{s a}+\varepsilon_{s a}$. The value of the slack variable is penalized in the objective function with a large coefficient so that the estimator minimizes $\varepsilon_{s a}$ first to obtain a feasible set.

\section{B. Phase 2: Robust Position Estimation}

In the second phase, the objective is to further reduce the impact of distorted path information in localization. The result of the first phase provides a coarse estimated area of the sensor position. Next, we deploy a robust position estimator to determine the final position within the approximated area. For each sensor $s \in \mathcal{S}$, its estimated position $\left(x_{s}, y_{s}\right)$ is the 


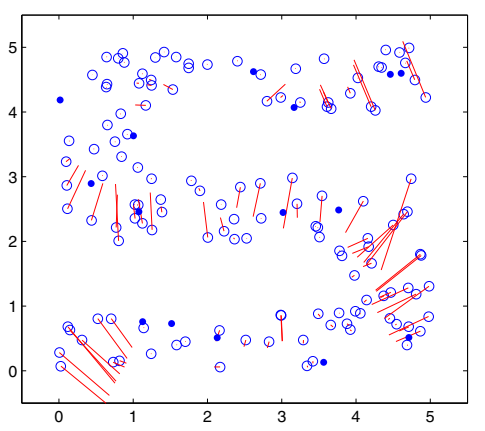

(a)

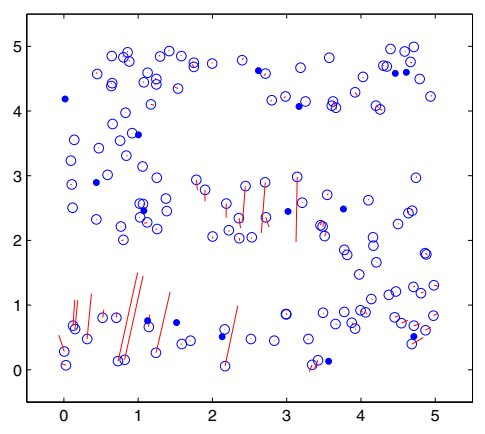

(b)

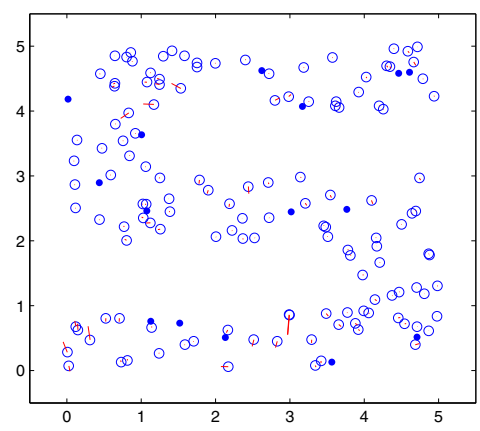

(c)

Fig. 3. Localization result comparison for $10 \%$ anchors in S-shaped topologies: (a) i-Multihop algorithm [15]: Avg_err $=0.4277 R$; (b) four nearest anchors algorithm [16]: Avg_err = 0.2827R; (c) two-phase algorithm: Avg_err =0.2297R .

solution of the following constrained least-penalty problem:

$$
\begin{aligned}
& \operatorname{minimize} \sum_{a \in \mathcal{A}} \phi(r(s, a))+\omega \sum_{a \in \mathcal{A}} \varepsilon_{s a}, \\
& \text { subject to }\left(x_{s}, y_{s}\right) \in P_{s},
\end{aligned}
$$

where $\omega$ is a large coefficient to penalize the slack variables and the residual function $r(s, a)$ is defined as

$$
r(s, a)=\widehat{d}_{s a}+\varepsilon_{s a}-d_{s a},
$$

where $d_{s a}$ is the Euclidean distance between the estimated position of sensor $s$ and anchor $a$

$$
d_{s a}=\sqrt{\left(x_{s}-x_{a}\right)^{2}+\left(y_{s}-y_{a}\right)^{2}},
$$

and the penalty function $\phi(r)$ is given by [19]

$$
\phi(r)= \begin{cases}\lambda r^{2}, & \text { if }|r|<\delta \\ \ln (|r|-\alpha)-\beta, & \text { otherwise }\end{cases}
$$

where

$$
\begin{gathered}
\alpha=\delta-\frac{1}{2 \lambda \delta}, \\
\beta=\ln \left(\frac{1}{2 \lambda \delta}\right)-\lambda \delta^{2},
\end{gathered}
$$

and $\lambda, \delta$ are parameters to be configured. In our simulations, $\lambda=80$ and $\delta=0.25$.

The robust position estimator in the two-phase algorithm has two features: (a) it increases the weight on measurements with residuals smaller than a threshold; (b) it reduces the emphases on erroneous measurements with large residuals. Compared with the least-squares estimator, the penalty function (4) has a steep slope when the residual is close to zero but increases much slower when the residual is large. The marginal penalty diminishes as the residual increases. Such differences result in that instead of minimizing the squared residuals of all measurements, the two-phase algorithm can fit the distances based on only reliable measurements achieving a better estimated position.

\section{Performance Evaluation}

In this section, we evaluate the performance of our proposed two-phase algorithm and compare it with two other existing multihop-based schemes: the four nearest anchors algorithm [16] and the i-Multihop: hybrid approach algorithm [15] in irregular areas. Two types of irregular topologies (C-shaped and S-shaped) are considered in our simulations. Let $R$ denote the communication range of the nodes. The deployment area is a $5 \times 5$ square area, where $R$ is normalized to one. Anchors are assumed to have the same communication range as sensors. 130 nodes are randomly distributed in C-shaped topologies. 150 nodes are distributed in S-shaped topologies to maintain the same average network connectivity level, i.e., average node degree. The performance metric is the normalized localization error, which refers to the distance from the estimated position to the true position of the sensor normalized with respect to the communication range $R$. In each set of simulation run, 50 trials with randomly generated topologies are performed and $95 \%$ confidence intervals are plotted in the figures.

We begin by comparing our proposed two-phase algorithm with the i-Multihop algorithm [15] and the four nearest anchors algorithm [16] in terms of the average localization error of all sensors in S-shaped topologies with a fixed proportion of anchors. It is assumed that the range measurements between pairwise nodes are accurate enough. Thus, the difference between the shortest path length and the Euclidean distance is the only source of error. Results with anchor density of 10\% are shown in Fig. 3, where the true positions of sensors are plotted by circles, the anchors are represented by dots and the localization error is drawn by lines. We can see that sensors on the edge have large errors for both the iMultihop algorithm and the four nearest anchors algorithm. For the i-Multihop algorithm, the reason for the large error is that sensors on the edge cannot be tightly bounded by the intersection areas. The distorted measurements push the estimated positions towards the boundaries of the intersection areas causing large errors. This can be seen directly from Fig. 3(a) where sensors on the edge have significant large errors. As for the four nearest anchor algorithm, the error is mainly due to the fact that the shortest paths to the four nearest 


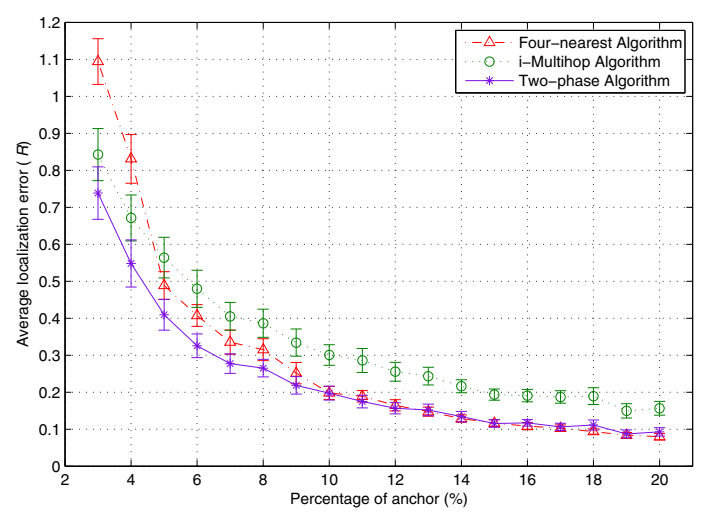

(a)

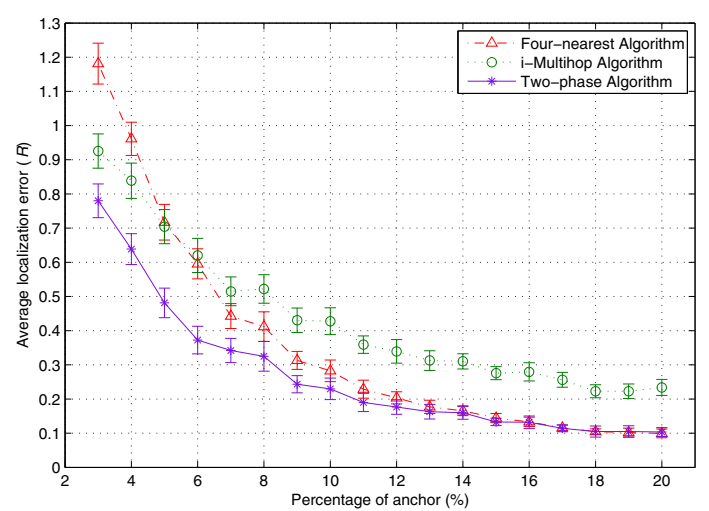

(b)

Fig. 4. Comparison between the two-phase algorithm, the four nearest anchors algorithm [16] and the i-Multihop [15] algorithm in C-shaped (a) and $S$-shaped topologies (b). The percentage of anchor varies from $3 \%$ to $20 \%$.

anchors are not always non-distorted as well as the position ambiguity problem which happens when the four anchors are placed closely on a line. More measurements which could be used to correct the position are disregarded in the four nearest anchors algorithm. In contrast, we can see that most of the sensors including those on the edge are located as their true positions in the two-phase algorithm. With $10 \%$ anchors, the two-phase algorithm can reduce the average localization error by $\frac{0.2827 R-0.2297 R}{0.2827 R} \times 100 \%=18.75 \%$ compared with the four nearest anchors algorithm in S-shaped topologies.

We analyze the sensitivity of anchor density on the localization error in irregular areas. Using randomly generated topologies, the average localization error results when the percentage of anchor varies from $3 \%$ to $20 \%$ in C-shaped and $\mathrm{S}$-shaped topologies are shown in Fig. 4. We can see that, for all three algorithms, the average localization error decreases as more anchors are available. The two-phase algorithm outperforms the other two algorithms in both topologies. In Cshaped topologies, the two-phase algorithm has a significant improvement in the accuracy when the anchor density is lower than $10 \%$. When the number of anchors increases, the average localization error converges to a fixed level and the confidence intervals of the four nearest anchors algorithm and the two-

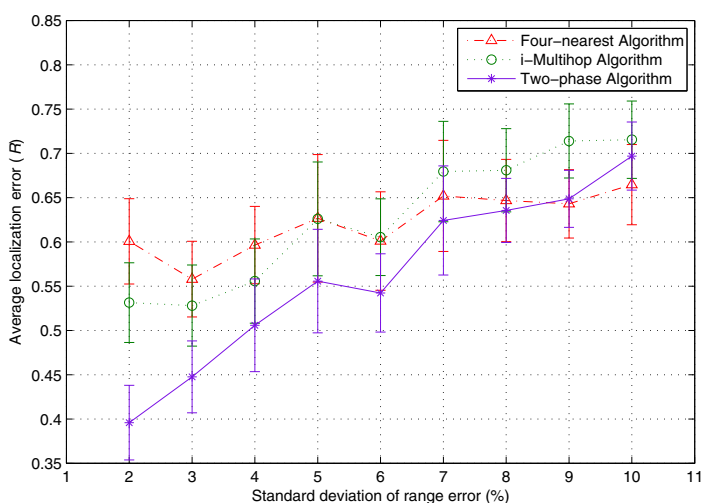

(a)

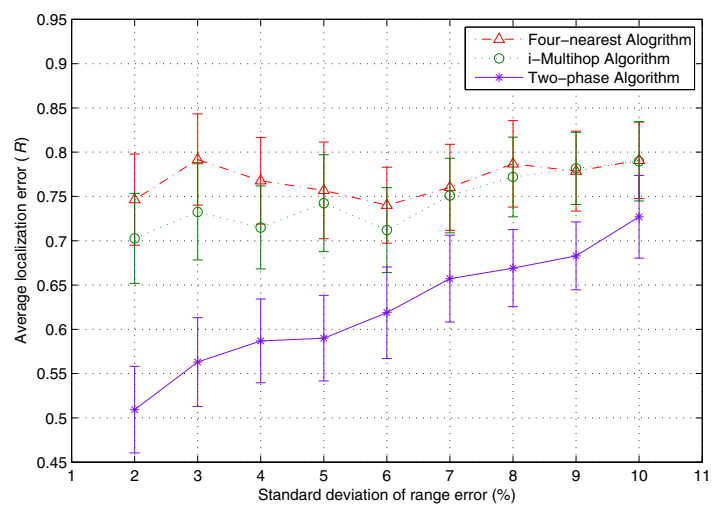

(b)

Fig. 5. Comparison between the two-phase algorithm, the four nearest anchors algorithm [16] and the i-Multihop [15] algorithm in C-shaped (a) and $S$-shaped topologies (b). The standard deviation of range error varies from $2 \%$ to $10 \%$.

phase algorithm overlap. This is because when the anchor density is higher than a threshold, most of the sensors have more than three anchors with line-of-sight paths. The i-Multihop algorithm does not perform well when the anchor density is high since it is unable to produce a good estimate when the intersection area is large. In S-shaped topologies, the average localization error is higher than that in C-shaped topologies for the same anchor density. Notice that the anchor density threshold for the average localization error to converge in Sshaped topologies is higher than that in C-shaped topologies showing that more anchors are needed in S-shaped topologies to achieve the same localization performance.

Next, we investigate the effect of range error on the localization performance of the two-phase algorithm. To model the range errors, we add a zero mean gaussian noise with standard deviation of $\sigma$ to the range measurement between neighboring nodes, i.e., $\hat{d_{i j}}=d_{i j}\left(1+N\left(0, \sigma^{2}\right)\right)$. The simulation results with anchor density of $5 \%$ when the standard deviation varies from $2 \%$ to $10 \%$ in $\mathrm{C}$-shaped and $\mathrm{S}$-shaped topologies are presented in Fig. 5. It can be seen from the figures that the twophase algorithm outperforms the other two algorithms when the standard deviation is below $9 \%$ in C-shaped topologies. In S-shaped topologies, the two-phase algorithm has the lowest 


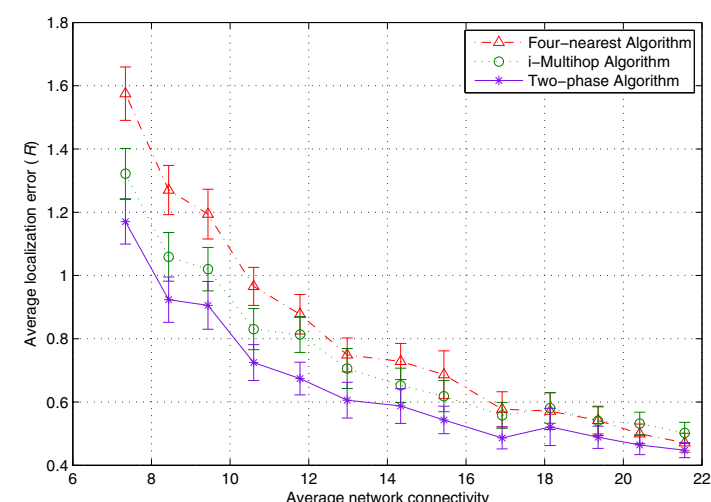

Fig. 6. Comparison between the two-phase algorithm, the four nearest anchors algorithm [16] and the i-Multihop [15] algorithm in C-shaped topologies with $5 \%$ anchors. Range error is $5 \%$. The average network connectivity level varies from 7.3 to 21.6 .

average localization error. Note that the four nearest anchors algorithm can perform better than the two-phase algorithm when the standard deviation is large because it uses only the four nearest anchors. In contrast, the error in the twophase algorithm can be accumulated to a large value in a multihop manner. Thus, ranging techniques with high accuracy but short range (e.g., ultrasonic) are suitable for the two-phase localization algorithm.

Finally, we consider the case where the communication range of the nodes varies. When the total number of nodes is fixed, a larger communication range results in a higher network connectivity level. To evaluate the performance under different connectivity levels, we modify the communication range from 0.6 to 1.2 in $\mathrm{C}$-shaped topologies to obtain an average network connectivity from 7.3 to 21.6. The anchor density is $5 \%$ and the standard deviation of range error is $5 \%$. The simulation result is shown in Fig. 6. We can see that the average localization error for all three algorithms decreases as the network connectivity level increases. The twophase algorithm outperforms the other two algorithms. The average localization error converges when the connectivity is high indicating that other sources of error (e.g., anchor density, range error) are the bottleneck of the performance.

\section{CONCLUSION}

In this paper, we studied the problem of locating sensors in irregular areas, where the shortest path distances can deviate significantly from the Euclidean distances. While the existing methods in the literature are not capable of removing the impact of irregularities thoroughly, we proposed a twophase algorithm to locate sensors in irregular areas. The twophase algorithm first identifies the approximated area of the sensor and then deploys a robust position estimator, which can eliminate the erroneous measurements. The estimated position is obtained by solving a least-penalty problem with communication range constraints. Through the two phases, the impact of distorted paths is minimized. We extended the original multihop localization scheme to irregular areas with desirable accuracy, which is necessary in real-world deployments. Simulation results showed that the two-phase algorithm provided better performances when compared with some of the previously proposed multihop algorithms in terms of the average localization error in both C-shaped topologies and S-shaped topologies. Future work includes applying the two-phase algorithm in a range-free setting.

\section{ACKNOWLEDGEMENT}

This research is supported by the Natural Sciences and Engineering Research Council (NSERC) of Canada under grant number 364962-08.

\section{REFERENCES}

[1] Y. Ho and N. H. Vaidya, "Location-aided routing (LAR) in mobile ad hoc networks," Wireless Networks, vol. 6, pp. 307-321, July 2000.

[2] D. Niculescu and B. Nath, "Ad hoc positioning system (APS)," in Proc. of IEEE Globecom, San Antonio, TX, Nov. 2001.

[3] Y. Shang, W. Ruml, Y. Zhang, and M. Fromherz, "Localization from connectivity," IEEE Trans. on Parallel and Distributed Systems, vol. 15, pp. 1-14, Oct. 2004.

[4] P. Biswas, T. C. Liang, K. C. Toh, T. C. Wang, and Y. Ye, "Semidefinite programming approaches to sensor network localization with noisy distance measurements," IEEE Trans. on Automation Science and Engineering, vol. 3, pp. 360-371, Oct. 2006.

[5] A. Savvides, H. Park, and M. B. Srivastava, "The n-hop multilateration primitive for node localization problems," Mobile Networks and Applications, vol. 8, pp. 443-451, 2003.

[6] B. Xiao, H. Chen, and S. Zhou, "Distributed localization using a moving beacon in wireless sensor networks," IEEE Trans. on Parallel and Distributed Systems, vol. 19, pp. 587-600, May 2008.

[7] F. Chan and H. C. So, "Accurate distributed range-based positioning algorithm for wireless sensor networks," IEEE Trans. on Signal Processing, vol. 57, pp. 4100 - 4105, Oct. 2009.

[8] R. Peng and M. L. Sichitiu, "Angle of arrival localization for wireless sensor networks," in Proc. of IEEE SECON, Reston, VA, Sept. 2006.

[9] K. Chintalapudi, A. Dhariwal, R. Govindan, and G. Sukhatme, "Ad-hoc localization using ranging and sectoring," in Proc. of IEEE Infocom, Hong Kong, China, Mar. 2004.

[10] V. Vivekanandan and V. W. S. Wong, "Concentric anchor beacon localization algorithm for wireless sensor networks," IEEE Trans. on Vehicular Technology, vol. 56, pp. 2733-2744, Sept. 2007.

[11] Y. Wang, X. Wang, D. Wang, and D. P. Agrawal, "Range-free localization using expected hop progress in wireless sensor networks," IEEE Trans. on Parallel and Distributed Systems, vol. 20, pp. 1540-1552, Oct. 2009.

[12] A. S. Paul and E. A. Wan, "RSSI-based indoor localization and tracking using sigma-point Kalman smoothers," IEEE Journal of Selected Topics in Signal Processing, vol. 3, pp. 860-873, Oct. 2009.

[13] Y. T. Chan, W. Y. Tsui, H. C. So, and P. C. Ching, "Time-of-Arrival based localization under NLOS conditions," IEEE Trans. on on Vehicular Technology, vol. 55, pp. 17-24, Jan. 2006.

[14] N. B. Priyantha, "The cricket indoor location system," Ph.D. dissertation, Massachusetts Institute of Technology, Jun. 2005.

[15] C. Wang and X. Li, "Sensor localization in concave environments," ACM Trans. on Sensor Networks, vol. 4, pp. 1-31, Jan. 2008.

[16] Y. Shang, H. Shi, and A. Ahmed, "Performance study of localization methods for ad hoc sensor networks," in Proc. of IEEE Int'l Conference on Mobile Ad Hoc and Sensor System (MASS), Fort Lauderdale, FL, Oct. 2004.

[17] B. Xiao, L. Chen, Q. Xiao, and M. Li, "Reliable anchor-based sensor localization in irregular areas," IEEE Trans. on Mobile Computing, vol. 9, pp. 60-72, Jan. 2009.

[18] S. Boyd and L. Vandenberghe, Convex Optimization. Cambridge University Press, 2004, p. 298.

[19] H. Kung, C. K. Lin, T. H. Lin, and D. Vlah, "Localization with snapinducing shaped residuals: Coping with errors in measurement," in Proc. of ACM MobiCom, Beijing, China, Sept. 2009.

[20] L. Doherty, K. Pister, and L. El Ghaoui, "Convex position estimation in wireless sensor networks," in Proc. of IEEE Infocom, Anchorage, Alaska, Apr. 2001.

[21] P. J. Huber, Robust Statistics. Wiley-Interscience, 2004. 